\title{
Evaluation of a Fourier Watermarking Method Robustness to Cards Durability Attacks
}

\author{
Rabia Riad ${ }^{1,2}$, Mohamed El Hajji ${ }^{1}$, Hassan Douzi ${ }^{1}$, \\ Rachid $\mathrm{Harba}^{2}$, and Frédéric Ros ${ }^{2}$ \\ 1 IRF-SIC Laboratory, Ibn Zohr University, BP 8106-Cité Dakhla, \\ 80000 Agadir, Morocco \\ 2 PRISME Laboratory,University of Orléans,12 Rue de Blois, 45067 Orleans, France
}

\begin{abstract}
In the context of an industrial application for securing identity cards, image watermarking is used to verify the integrity and authenticity of images printed on plastic cards support. In this area the watermark must survive image modifications related to print-and-scan process and the degradations submitted by ID cards through its lifetime. In this work various card durability attacks were studied (bending, scratches, color fading) as well as some additional other attacks that reflects other possible attacks in the given industrial context. The ID images were watermarked by a new Fourier watermarking method based on print/scan counterattacks (blurring correction and colors restorations) that allows improvement of the robustness of the watermarking method. Results show a noticeable increase of the overall performances of the Fourier watermarking method against durability attacks that is suitable in the context of the industrial application of interest.
\end{abstract}

Keywords: cards durability; print/scan; DFT watermarking; counterattack; ID images.

\section{Introduction}

Security is one of the highest challenges of access control system based on Smart cards. The development of technology and image processing play a major role in security process. In this context, digital watermarking is used to verify the integrity and authenticity of a portrait of the holder, printed on cards support 12 .

Digital watermarking is information intelligently embedded in the host data such that an unauthorized party cannot use this document [34], The specification of watermarking schemes depends on their usage scenario and there is no ideal solution able to match with all requirements with $100 \%$ of efficiency while being fast, secure, imperceptible and robust. For ID card applications, the essentials requirements for digital watermarking are the robustness to printing and scanning attacks, and the robustness to the cards durability attacks.

Cards durability is a key factor in ID card application field. Indeed, the cards and ID images must be resistant to both normal and extreme usage conditions

A. Elmoataz et al. (Eds.): ICISP 2014, LNCS 8509, pp. 280-288, 2014.

(C) Springer International Publishing Switzerland 2014 
such as being left in an overheated parked car or bent in a wallet or pocket. Cards durability groups all possible aggressions that can be submitted by the card along its lifetime (bending, scratches, and color fading) [1. The watermarks embedded in the ID images do not have to be altered through the ID card lifetime, which can be up to 10 years [7. Improvements in performance of watermarking schemes can be then obtained by understanding the attacks to select the most appropriate domain insertion and optimize the embedding and retrieval processes. Several studies have been launched to develop and look at the influences of various overlays and core films used for card production on the color changes over time due to the ageing of the cards [5]. In [6], they showed that the choice of card body material can influence the available options for printing and finishing, which can affect the eventual durability of the finished cards. However, there is a lack of study of the influence of durability attacks on watermarked image.

This paper is interested in the capability of watermarking techniques to protect pictures printed on plastic card and the resistance of the watermark to the print/scan and durability attacks. In order to attest the robustness of the watermark to these attacks, a series of degradations have been established to simulate durability attacks. In this work, series of degradations that reflects the cards durability were applied to the printed/scanned images. Additional attacks are used also to evaluate the robustness of the watermark such as JPEG compression, additive Gaussian white noise AGWN, histogram equalization and multiple watermarks. The watermark is embedded in ID images using a new watermarking method based on Fourier transform and print-and-scan counterattacks. These counterattacks are within two stage, blurring corrections and color restoration. The details of the watermarking method are already accepted in [8]. This paper will thus provide only the outline of the method.

The paper is organized as follow: section 2 describes the attacks related to the card durability and additional attacks. Section 3 presents briefly the proposed watermarking method and print-and-scan counterattacks. Section 4 shows the experimental results. The conclusion is described in the final section.

\section{Card Durability Attacks and Additional Attacks}

The watermark is embedded in the digital image of ID card, and then it can be extracted from the physical ID by scanning the image [2]. As shown in Fig [1] the input image is introduced into the watermarking process with the watermark and other watermarking parameters. The watermarked image is subjected to natural degradation linked the print/scan attacks and the card durability. The block of attacks includes attacks studied after the print/scan operation that simulates ID card durability. There are many kinds of durability attacks such as color fading, dust, and scratches... All these attacks appear during the use of the card and may prevent the marks detection during the integrity verification or authentication of the ID card.

Recently in [8], we have proposed a watermarking method which consists on pretreatments that places the image in a favorable context for the mark 


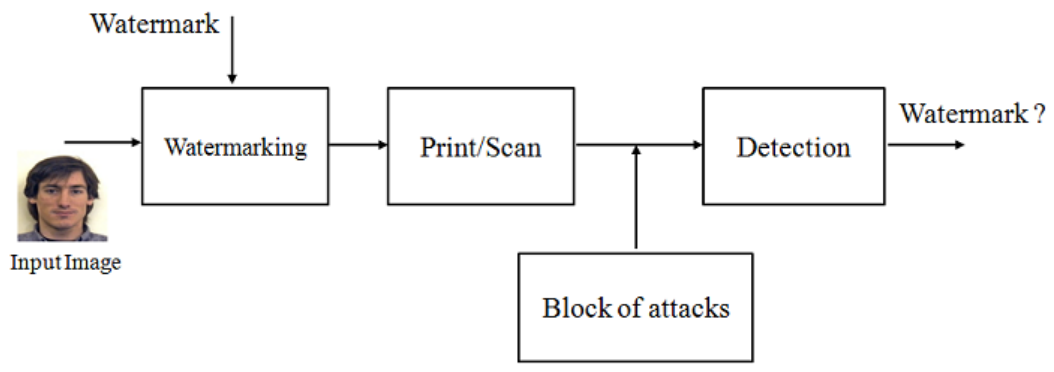

Fig. 1. Watermarking and detection process

extraction. In which, we studied its behavior against the Print/Scan attack. In this work, we show the advantages of this watermarking method and their main limitations against card durability. This section, described the most important attacks linked to cards durability.

\subsection{Card Durability Attacks}

Most ID card personalization features such as photos, text, logos, bar codes. . ., are regularly exposed to a variety of potentially destructive elements that can degrade printed images [6]. Essentially, secure documents do not have to be altered through the ID document lifetime, which can be up to 10 years. ID documents also promote the image of the issuing authority and it is important that the document does not need to be reissued before its expected end of validity [7].

In this work, the print-and-scan attacks are completely integrated into the watermarking process. The card durability is simulated by a series of attacks that can be classified in two categories: mechanical and photometrical.

Mechanical Attacks: The most important degradation of card durability is the amount of times the card is inserted into a reader. This can be a result of persistent bending or flexing of the card, this degradation leads to the characteristic "barrel" shape. The barrel distortion model [9] is:

$$
a_{u}=a_{d}\left(1+k a_{d}^{2}\right)
$$

where $a_{u}$ and $a_{d}$ are radial distances in an undistorted and in a distorted image respectively, and $k$ is the distortion parameter.

The card and ID image will also suffer from dust, scratches, or artifacts due to the non-protected use of the card. This kind of attacks can be simulated by:

1. Adding an impulsive noise;

2. Adding lines randomly in the image: It is not possible to simulate all the dimensions, sizes, and orientations of image scratches. That is why we decided to draw various verticals lines randomly in the image to simulate this type of deterioration; 
3. Removed random parts from the images: This modification of the image results in removal of part of the image. We observe a hole in the image. The tests performed are based on cut a square of dimensions $N \times N$ pixels of the image. This test is performed with different sizes of cutting and cutting different places;

Fig 2 shows the results of this type of attacks on an image.

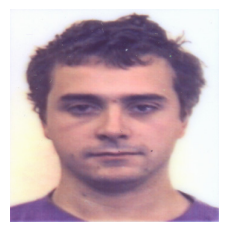

(a)

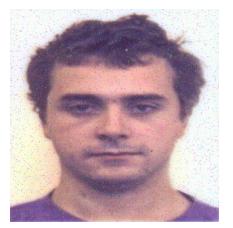

(b)

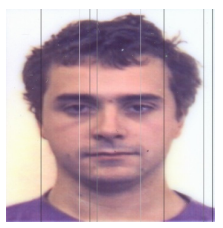

(c)

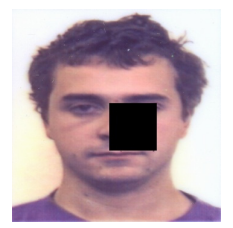

(d)

Fig. 2. Example of mechanical attacks, Original image (a), Impulse noise (b), 8 lines added (c), $5 \%$ removed from the image (d)

Photometrical Attacks: A phenomenon of color fading (or photo-degradation) occurs when the card is exposed to sunlight for a long time. This degradation introduces a saturation decreasing and hue modification of ID images. This degradation can be delayed by different means (e.g. film UV protection on the image), but there will always be a color change. The simulation of color fading consists in converting the image from RGB to HSI color space then decreasing through the saturation value, increasing the hue value and converting back the image to RGB color space. Fig 3 shows the result of this type of the attack.

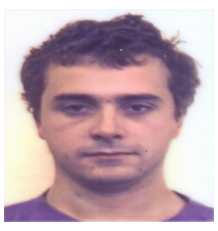

(a)

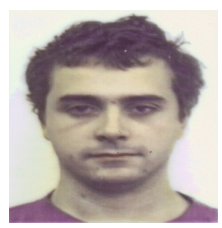

(b)

Fig. 3. Original image (a) and image after color fading (b)

\subsection{Additional attacks}

In other cases, the watermark must survive degradations, after print-and-scan process, due to the transmission process such as additive noise, malicious manipulations and unintentional processing.

In this work, the robustness of the watermark is evaluated using some kinds of unintentional manipulations such as JPEG compression, additive Gaussian 
noise, and some malicious manipulations such as histogram equalization, rotation and multiple watermarking. The last attack consists in embedding another watermark to the printed and scanned images using a blind spatial watermarking as described in 3 . The strength of watermarking was varied in order to reach the limit of the first watermark robustness. Fig 4 shows the results of this type of the attacks.

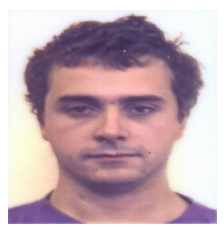

(a)

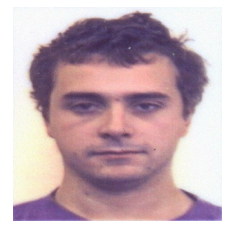

(b)

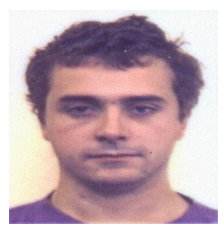

(c)

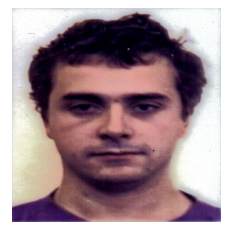

(d)

Fig. 4. Example of additional attacks, Original image (a), AGWN noise (b), Multiple watermarking (c), Histogram equalization (d)

\section{Watermarking Method and Print-and-Scan Counterattacks}

\subsection{Watermarking Method}

According to 1011, the watermark is embedded in ID image using a watermarking method based on Fourier transform. The embedding process consists on the following steps: First, luminance values of the cover work are transformed in the Fourier domain. Then the watermark as a pseudo-random sequence of $N$ binary elements is inserted in the magnitude coefficients along a circle of radius $r$, which can be expressed with this equation:

$$
M_{w}(x, y)=M_{0}(x, y)+\alpha \times W(x, y)
$$

where $M_{w}$ is the magnitude of the watermarked DFT coefficient, $M_{0}$ is the original one, $\alpha$ is the strength parameter, and $(x, y)$ are image coordinates in the frequency domain.

Finally, the colored watermarked image is reconstructed by applying the inverse DFT to obtain the luminance of the watermarked image from which color image is recovered using the unmodified chrominance components. The peak signal-to-noise ratio (PSNR) is used to evaluate the quality of watermarked images. PSNR values above $40 \mathrm{~dB}$ indicate almost invisible degradation and values below $30 \mathrm{~dB}$ indicate high degradation 12 . For these reasons, $40 \mathrm{~dB}$ is chosen here to watermark images.

In the detection process a blind detector scheme is performed using only the watermarked image and the watermark $W$. Before watermark detection, the DFT is applied to image luminance. Fourier coefficients are extracted from the magnitude along the radius $r$. The normalized cross-correlation is computed 
between the extracted coefficients $F$ and the sequence $W$ of the watermark. As a rotation could occur during the print-and-scan process, the maximum of the normalized cross-correlation $C_{M a x}$ is to be estimated as follow:

$$
C_{M a x}=\operatorname{Max}_{1<j<N}\left(\frac{\sum_{i=0}^{N-1}(W(i)-\bar{W})(F(i+j)-\bar{F})}{\sqrt{\sum_{i=0}^{N-1}(W(i)-\bar{W})^{2} \sum_{i=0}^{N-1}(F(i+j)-\bar{F})^{2}}}\right)
$$

where $N$ is the sequence length, $\bar{W}$ and $\bar{F}$ are the mean of the watermark and extracted Fourier coefficients, respectively.

If the maximum value of the normalized cross-correlation exceeds a threshold $t$, the watermark is considered as detected; else the watermark is not detected. To avoid the false positive detection for a given database, the threshold value $t$ is chosen greater than the highest detection value of the unwatermarked images [1].

\subsection{Print-and-Scan Counterattacks}

The print-and-scan attack reduces the information contained in the high frequencies of an image, and produces images with different colors from the original ones. The best defense to this process is to counterattack. The print-and-scan attacks are considered as a linear filter with additive noise, which introduces blurring in the output images, in addition to a non-linear function that lead to the color distortions.

The Wiener filter is used for counterattack image blurring, since the print-andscan channel parameters can be assessed. This filter is expressed in the frequency domain by:

$$
F=G \frac{H^{*}}{|H|^{2}+\frac{1}{S N R}}
$$

$F$ and $G$ are the Fourier transforms of the corrected and degraded images, respectively. And $H$ represents the Fourier transform of the impulse response $h$, $H^{*}$ is the complex conjugate of $H$. The SNR (signal to noise ratio) is equal to $P / P_{n}$, with $P$ and $P_{n}$ are the power of the image and the power of the noise, respectively. The Wiener filter requires the impulse response $h$ and the SNR which can be measured from the print-and-scan channel.

For color distortions counterattack, the non linear color transfer functions are computed for R, G and B color components. The first step consists in printing and scanning 246 colors chosen to be representative of the colors in ID images, especially skin and hair colors. The transfer functions of the RGB components are estimated using a 4-order polynomial curve [13. The inverse functions can be estimated from which 1D lookup tables (LUT) are created. Finally the lookup tables are used for restoring color in printed and scanned images. 


\section{Results}

To evaluate the robustness of the watermark to card durability and additional attacks, 100 ID images are selected from PICS databases 14. The images were watermarked then printed on plastic card support using a card printer Fargo Persona C25. Printed images were scanned using a flatbed scanner HP ScanJet. The series of degradations described previously were applied to the 100 watermarked and 100 non-watermarked images after the print-and-scan process.

Table 1. Detection rate for a fixed threshold $(t=0.3)$ after various attacks

\begin{tabular}{|c|c|c|}
\hline Attacks & Without counterattacks & With counterattacks \\
\hline Barrel distortion $(k=0.01)$ & $92 \%$ & $100 \%$ \\
\hline Barrel distortion $(k=0.02)$ & $81.5 \%$ & $96 \%$ \\
\hline Barrel distortion $(k=0.03)$ & $71.5 \%$ & $83.5 \%$ \\
\hline Barrel distortion $(k=0.04)$ & $58.5 \%$ & $67.5 \%$ \\
\hline 2 lines added & $93 \%$ & $99 \%$ \\
\hline 4 lines added & $92.5 \%$ & $99 \%$ \\
\hline 8 lines added & $91 \%$ & $97 \%$ \\
\hline 16 lines added & $84.5 \%$ & $83.5 \%$ \\
\hline Impulsive noise $2 \%$ & $57.5\left(75.5^{1}\right) \%$ & $52.5\left(97.5^{1}\right) \%$ \\
\hline $1 \%$ removed from the image & $84 \%$ & $99.5 \%$ \\
\hline $2 \%$ removed from the image & $83 \%$ & $99 \%$ \\
\hline $3 \%$ removed from the image & $81 \%$ & $99 \%$ \\
\hline $4 \%$ removed from the image & $79 \%$ & $97.5 \%$ \\
\hline $5 \%$ removed from the image & $67 \%$ & $95.5 \%$ \\
\hline Color Fading & $87.5 \%$ & $99 \%$ \\
\hline JPEG $(90 \%)$ & $93 \%$ & $100 \%$ \\
\hline JPEG $(80 \%)$ & $89 \%$ & $99.5 \%$ \\
\hline JPEG $(70 \%)$ & $83.5 \%$ & $99.5 \%$ \\
\hline JPEG $(60 \%)$ & $78 \%$ & $98.5 \%$ \\
\hline JPEG $(50 \%)$ & $74 \%$ & $95.5 \%$ \\
\hline JPEG $(40 \%)$ & $66 \%$ & $91.5 \%$ \\
\hline $\operatorname{AGWN}\left(\sigma^{2}=6.5,40 \mathrm{~dB}\right)$ & $91\left(91^{2}\right) \%$ & $98.5\left(99.5^{2}\right) \%$ \\
\hline $\operatorname{AGWN}\left(\sigma^{2}=20,35 \mathrm{~dB}\right)$ & $88.5\left(88.5^{2}\right) \%$ & $96.5\left(99.5^{2}\right) \%$ \\
\hline AGWN $\left(\sigma^{2}=65,30 \mathrm{~dB}\right)$ & $81.5\left(82^{2}\right) \%$ & $72\left(94^{2}\right) \%$ \\
\hline Multiple Watermarks(40dB) & $87.5 \%$ & $95 \%$ \\
\hline Multiple Watermarks $(35 \mathrm{~dB})$ & $81 \%$ & $88.5 \%$ \\
\hline Multiple Watermarks(30dB) & $70.5 \%$ & $69 \%$ \\
\hline Histogram equalization & $79 \%$ & $87.5 \%$ \\
\hline Rotation $30^{\circ}$ & $79.5 \%$ & $97 \%$ \\
\hline Rotation $-30^{\circ}$ & $80 \%$ & $98.5 \%$ \\
\hline
\end{tabular}

The detection rate was determined after images were subjected to an attack. The value of the detection threshold was set to $t=0.3$. This value was chosen

\footnotetext{
${ }^{1}$ Median filter $(3 \times 3)$.

${ }^{2}$ Gaussian filter.
} 
because the highest detection value of the non-watermarked set of images was 0.28 , thus this reduces false positive detection for the given dataset.

Test results are listed in Table 1, the first column gives the name of attacks and their parameters. The second column presents the detection rate for the fixed threshold. The third depicts the detection rate using the print-and-scan counterattacks. However, results shows for the most cases of attacks that the print-and-scan counterattacks improve the detection rate. We have observed that the proposed counterattacks have negative impact on impulsive noise and AGWN noise. This negative impact can be explained by the fact that images deconvolution in general increases high frequencies corresponding to the noise. To reduce noises we apply two kinds of filters; Median filter for impulsive noise and Gaussian filter for AGWN noise. Results show an improvement of the method performance.

\section{Conclusion}

This paper presents an evaluation of watermark robustness to the print-andscan and card durability attacks in the context of an industrial application that concerns securing identity images printed on plastic medium. To deal with printand-scan attacks, print-and-scan counterattacks were applied before the detection step within 2 stages; blurring corrections and color restoration. A series of degradations have been established to simulate the aggressions submitted by ID cards. These attacks are applied to printed and scanned images, in order to confirm the robustness of the watermark to the card durability. Results shows that the print-and-scan counterattacks improved the detection rate for most attacks. Moreover, the method is fast and simple and thus can be used in the context of securing images printed on ID cards.

Acknowledgments. This work is supported by the Franco-Moroccan Volubilis Project 2697WA (PHC MA/12/279) and by Gemalto coseqID contract $N^{\circ}$ : SUREO13090.

\section{References}

1. Ros, F., Borla, J., Leclerc, F., Harba, R., Launay, N.: An industrial watermarking process for plastic card supports. In: Proc. IEEE ICIT, pp. 2809-2814 (2006)

2. Picard, J., Vielhauer, C., Thorwirth, N.: Towards fraud-proof ID documents using multiple data hiding technologies and biometrics. In: Proc. SPIE, vol. 416, pp. 416-427 (2004)

3. Cox, I.J., Miller, M.L., Bloom, J., Fridrich, J., Kalker, T.: Digital watermarking and steganography, 2nd edn. Morgan Kaufmann (2007)

4. El Hajji, M., Douzi, H., Harba, R., Mammass, D., Ros, F.: New image watermarking algorithm based on mixed scales wavelets. JEI -SPIE 21, 13003-1-13003-7 (2012)

5. Fogra: The Fogra testing laboratory for ID-cards and passport documents, wWw. fogra.org 
6. Datacard Group: Durability of Smart Cards for Government eID. Part of a series of Datacard Group white papers for the secure document issuer

7. Karppinen, K., Dequidt, M.: Smart card interfaces and government applications. White paper, Gemalto Government Programs 06/08 (2008)

8. Riad, R., Harba, R., Douzi, H., Elhajji, M., Ros, F.: Print-and-scan counterattacks for plastic card supports Fourier watermarking. Accepted to IEEE Industrial Electronic Symposium in Istambul (2014)

9. Bailey, D.G.: A new approach to lens distortion correction. In: Proc. Image and Vision Computing, pp. 59-64 (2002)

10. Solachidis, V., Pitas, I.: Circularly symmetric watermark embedding in 2-D DFT domain. IEEE Transactions on Image Processing 10, 1741-1753 (2001)

11. Poljicak, A., Mandic, L., Agic, D.: Discrete Fourier transform-based watermarking method with an optimal implementation radius. J. Electron. Imaging 20(3), 033008-1-033008-8 (2011)

12. Cheddad, A., Condell, J., Curran, K., Kevitt, M.: Digital image steganography suvey and analysis of current methods. Signal Processing 90, 727-752 (2010)

13. Nakamura, S.: Applied Numerical Methods in C. Prentice Hall (1995)

14. database: PICS (Psychological Image Collection at Stirling) - Aberdeen (2012) 